\title{
LINEAR OPERATORS INEQUALITY FOR $n$-CONVEX FUNCTIONS AT A POINT
}

\author{
Josip PeČArić, Marjan Praljak And Alfred Witkowski
}

Abstract. We study necessary and sufficient conditions on linear operators $A$ and $B$ for inequality $A f \leqslant B f$ to hold for every function $f$ that is $n$-convex at a point.

Mathematics subject classification (2010): 26D99, $26 \mathrm{D} 15$.

Keywords and phrases: $n$-convex functions, $n$-convex functions at a point, Levinson's inequality.

\section{REFERENCES}

[1] I. A. BAloch, J. PeČArić And M. Praljak, Generalization of Levinson's inequality, J. Math. Inequal., to appear

[2] I. B. LACKOVIĆ AND P. M. VASIć, Notes on convex functions II: On continuous linear operators defined on a cone of convex functions, Univ. Beograd. Publ. Elektrotehn. Fak. Ser. Mat. Fiz. No. 602633 (1978), 53-59.

[3] N. Levinson, Generalization of an inequality of Ky Fan, J. Math. Anal. Appl. 8 (1964), 133-134.

[4] A. MCD. MERCER, Short proof of Jensen's and Levinson's inequalities, Math. Gazette 94 (2010), 492-495.

[5] J. PeČArić, M. Praljak And A. Witkows Ki, Generalized Levinson's inequality and exponential convexity, Opuscula Math. 35, no. 3 (2015), 397-410.

[6] J. Pečarić, F. Proschan and Y. L. Tong, Convex Functions, Partial Orderings, and Statistical Applications, Academic Press Inc., San Diego, 1992.

[7] T. Popoviciu, Les fonctions convexes, Herman et cie, Paris, 1944.

[8] T. Popoviciu, Sur une generalisation des fonctions "spline", Math. Struct. Comp. Math.-Math. Modeling, Sofia (1975), 405-410.

[9] A. Witkowski, On Levinson's inequality, Ann. Univ. Paed. Cracov. Stud. Math. 12 (2013), 59-67. 